\title{
Key Concepts for Estimating the Burden of Surgical Conditions and the Unmet Need for Surgical Care
}

\author{
Stephen Bickler • Doruk Ozgediz • Richard Gosselin • \\ Thomas Weiser • David Spiegel · Renee Hsia • \\ Peter Dunbar · Kelly McQueen · Dean Jamison
}

Published online: 28 October 2009

(C) The Author(s) 2009. This article is published with open access at Springerlink.com

\begin{abstract}
Background Surgical care is emerging as a crucial issue in global public health. Methodology is needed to assess the impact of surgical care from a public health perspective.

Methods A consensus opinion of a group of surgeons, anesthesiologists, and public health experts was established regarding the methodology for estimating the burden of surgical conditions and the unmet need for surgical care.

Results For purposes of analysis, we define surgical conditions as any disease state requiring the expertise of a surgically trained provider. Abnormalities resulting from a surgical condition or its treatment are termed surgical sequelae. Surgical care is defined as any measure that reduces the rates of physical disability or premature death associated with a surgical condition. To measure the burden
\end{abstract}

\author{
S. Bickler $(\bowtie)$ \\ Division of Pediatric Surgery, University of California \\ at San Diego, San Diego, USA \\ e-mail: sbickler@ucsd.edu \\ D. Ozgediz \\ Division of Pediatric Surgery, University of Toronto, \\ 555 University Avenue, Toronto, ON, Canada M5G 1X8 \\ R. Gosselin \\ School of Public Health, University of California at Berkeley, \\ 50 University Hall \#7360, Berkeley, CA 94720-7360, USA \\ T. Weiser \\ Department of Health Policy and Management, Harvard \\ University, Kresge 4th Floor, 677 Huntington Avenue, \\ Boston, MA 02115, USA \\ D. Spiegel \\ University of Pennsylvania Children's Hospital of Philadelphia, \\ 34th Street and Civic Center Boulevard, Philadelphia, \\ PA 19104, USA
}

of surgical conditions and unmet need for surgical care we propose using cumulative disability-adjusted life-year (DALY) curves generated from age-specific populationbased data. This conceptual framework is based on the premise that surgically associated disability and death is determined by the incidence of surgical conditions and the quantity and quality of surgical care. The burden of surgical conditions is defined as the total disability and premature deaths that would occur in a population should there be no surgical care; the unmet need for surgical care is defined as the potentially treatable disability and premature deaths due to surgical conditions. Burden of surgical conditions should be expressed as DALYs and unmet need as potential DALYs avertable.

\footnotetext{
R. Hsia

University of California, Box 1377, 1001 Potrero Avenue 1E21,

San Francisco, CA 94143-1377, USA

P. Dunbar

Department of Anesthesia, University of Washington, PO Box 35640BB-1469, Health Sciences, 1959 NE Pacific Street, Seattle, WA 98195-6540, USA

K. McQueen

Harvard Humanitarian Initiative, Harvard University, 14 Story Street, 2nd Floor, Cambridge, MA 02138, USA

D. Jamison

Department of Global Health, University of Washington, Seattle, WA 98195, USA
} 
Conclusions Methodology is described for estimating the burden of surgical conditions and unmet need for surgical care. Using this approach it will be feasible to estimate the global burden of surgical conditions and help clarify where surgery fits among other global health priorities. These methods need to be validated using population-based data.

\section{Introduction}

Over the past several years there has been a growing interest in the role of surgery in global public health [1-4]. Inspired by the work of economist Dean Jamison [5] and the chapter "Surgery" in the Disease Control Priorities in Developing Countries, 2nd edition [6] on the cost-effectiveness of surgical care in the developing world, a Global Burden of Surgical Disease Working Group (GBSDWG) was formed in 2008 [7]. This group of surgeons, anesthesiologists, emergency physicians, and public health experts met for the first time in Seattle on April 16-17, 2008 to begin a dialogue that explores the role of surgery in public health, priority areas of research, and how best to advocate for support of global surgical programs.

The overwhelming consensus of attendees at this meeting pointed to a critical lack of data concerning the true portion of the global burden of diseases that could be alleviated by surgical expertise. Current methodology was inadequate for measuring the impact of surgical care. During these discussions, several key problems were identified that have impeded accurate measurement of the global burden of surgical conditions: First, basic definitions related to surgical conditions have not been clarified. Second, surgery is a procedure-oriented specialty; and previous global burden of diseases estimates have not been approached from an intervention perspective. Third, the large number of surgical diagnoses and treatments and wide range in patient ages complicate the analysis.

In this article, we propose definitions for assessing surgery from a public health perspective. We also describe a conceptual framework within which we can estimate the burden of surgical conditions and the unmet need for surgical care.

\section{Methods}

At the 2008 GBSDWG meeting, a committee was designated to work on surgical definitions. The committee included five surgeons, two anesthesiologists, an emergency room physician, and a health economist. All members of the committee had global health experience, and six members had advanced degrees in public health. Over the subsequent year, the committee worked together to develop a consensus opinion on definitions and methodology for assessing surgical care from a public health perspective.

\section{Results}

Table 1 summarizes our proposed definitions for analyzing surgical care from a public health perspective. The definitions were crafted with the recognition that not all surgical conditions require a procedure; nonphysicians or general doctors, rather than surgical specialists, often provide surgical care in low-income countries; and multiple surgical sequelae can result from a single surgical condition.

Two parameters are described for assessing the public health impact of surgical conditions and surgical care. The severity of a surgical condition is represented as a surgical disability weight (DW). As used in the Disease Control Priorities Project [8], surgical disability weight is a health state valuation expressed on a ratio scale between 0 (full health) and 1 (states equivalent to death).

To measure the impact of surgical care, we propose a parameter called the "value of surgical care" (VSC), which is the relative ability to prevent or reverse a surgical disability. In a manner similar to disability weight, the value of surgical care is expressed on a ratio scale (between 0 and 1) according to how effectively the intervention reverses the disability. Surgical care is curative when the value of surgical care matches the disability weight (VSC $=\mathrm{DW})$ or partially restorative when the value of surgical care is less than the disability weight (VSC $<\mathrm{DW})$. In situations where surgical care results in a disability greater than the initial condition, the complication is calculated as a separate surgical condition with its own disability weight.

The method we describe assigns a DALY ${ }^{1}$ value to each surgical condition and a DALY averted value to each surgical intervention. Using this approach, it is possible to measure quantitatively the burden of surgical conditions and the impact of surgical care.

Figure 1 presents a conceptual framework for estimating the burden of surgical conditions and the unmet need for surgical care. The framework is based on the premise that surgically related DALYs within a population are determined by the incidence of surgical conditions and the quantity and quality of surgical care. For analysis purposes, a cumulative incidence function of surgical DALYs is proposed. Cumulative surgical DALYs can be calculated from age-specific data (Appendix 1).

As shown in Fig. 1, average cumulative DALY curves can vary depending on the level of surgical care. In the best

\footnotetext{
${ }^{1}$ Disability adjusted life year. The DALY combines time lost due to premature mortality (years of life lost, or YLL) and time lived with the disability (years of healthy life lost due to disability, or YLD). Thus, DALY $=$ YLL + YLD.
} 
Table 1 Terminology for analyzing surgical care from a public health perspective

Surgical condition-disease state requiring the expertise of a surgically trained provider

Surgical sequelae-abnormalities that result from a surgical condition or its treatment.

Surgical disability - physical deficit associated with a surgical sequela

Surgical care-any intervention directed at reducing the disability or premature death associated with a surgical condition

Surgical procedure - the suturing, incision, excision, or manipulation of tissue; or other invasive procedure that usually, but not always, requires local, regional, or general anesthesia

Disability weight - measure of the relative valuations of a health state on an interval scale

Value of surgical care-measure of the relative ability to prevent or reverse a surgical disability

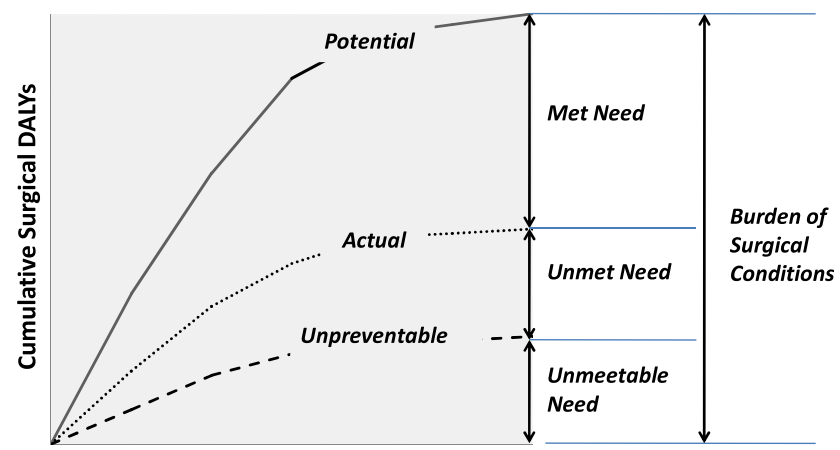

Age (years)

Fig. 1 Conceptual framework for estimating the burden of surgical conditions and the need for surgical care. Surgical disability and premature death in a population is a time-dependent function that relates to the incidence of surgical conditions and the quantity and quality of surgical care. The potential, actual, and unpreventable curves represent the age-specific cumulative disability-adjusted lifeyears (DALYs) with different levels of surgical care. See Table 2 for the definitions describing the need for surgical care and the burden of surgical conditions

case scenario (when surgical care is ideal), the only surgical disability and premature death in a population is that which is unpreventable. In the worst case scenario (when surgical care is nonexistent), surgical disability and death reaches the maximum possible. Because surgical care is never completely absent, nor ever ideal, the actual surgical DALY curve lies somewhere between these two curves.

Table 2 summarizes terminology for describing the need for surgical care within a population. The met need refers to the surgical care that has been provided, and unmet need refers to potentially treatable disability and death due surgical conditions. The unmeetable need is the disability and premature death that is unavoidable, even with the best surgical care. The burden of surgical conditions is defined as the total disability and premature death that would occur in a population should there be no surgical care. The burden of surgical conditions is expressed as DALYs, met need for surgical care as DALYs averted, and unmet need as potential DALYs avertable. Appendix 2 shows an example of how these parameters can be calculated using population-based data.

\section{Discussion}

There is an ongoing debate about the relative importance of surgical care in global public health. Definitions of the burden of surgical conditions and the impact of surgical care are critical to clarifying the relative priority of surgery within global health. To date, neither the global burden of surgical conditions nor the effect of surgical treatment has been quantified with existing measures [9]. As major disparities in surgical care exist between high and low-income countries, an estimate of regionally specific disabilityadjusted life-years that can be averted by surgical interventions is also needed [6].

In this article, we outline a conceptual framework for estimating the burden of surgical conditions and unmet need for surgical care. Our definition for surgical conditions is broader than the definition used in the Disease Control Priorities Project, where surgical conditions were defined as "any treatment that includes suture, incision, excision, manipulation, or other invasive procedure that

Table 2 Proposed terminology for describing and measuring burden of surgical conditions and need for surgical care

\begin{tabular}{lll}
\hline Term & Definition & Unit of measure \\
\hline Burden of surgical conditions & $\begin{array}{l}\text { The disability and premature death that would exist in a population } \\
\text { without any surgical care }\end{array}$ & DALYs \\
Met need for surgical care & $\begin{array}{l}\text { The disability and premature death in a population that has been } \\
\text { prevented or corrected with surgical care }\end{array}$ & DALYs averted \\
Unmet need for surgical care & $\begin{array}{l}\text { The disability and premature death in a population that is preventable } \\
\text { or correctable with surgical care }\end{array}$ & $\begin{array}{c}\text { DALYs potentially } \\
\text { avertable }\end{array}$ \\
Unmeetable need for surgical care & $\begin{array}{c}\text { The disability and premature death in a population that is unpreventable } \\
\text { or uncorrectable with even the best surgical care }\end{array}$ & DALYs unavertable
\end{tabular}

$D A L Y s$ disability-adjusted life-years 
usually, but not always, requires local, regional, or general anesthesia" [6]. The main consideration in broadening this definition is the fact that surgical conditions do not always require a surgical procedure. Examples include the care of most head injuries and nonoperative management of blunt abdominal injuries (e.g., splenic injury in a child). A clear advantage of the broader definition is that it more accurately reflects the surgical workload, which may be critical when limited resources are being allocated. While the ratio of nonoperative to operative surgical care is likely to vary by specialty, limited data suggest that it may be substantial. In a community-based study of pediatric surgical conditions in West Africa, only $46 \%$ of children presenting with a surgical condition required a surgical procedure [10]. Research is needed to determine what this ratio might be in other areas of surgery.

To be consistent with the latest Global Burden of Disease (GBD) study, we use the term surgical sequelae to describe any abnormality that results from a surgical condition or its treatment [11]. As used in the current GBD study, sequelae refer to the combination of health states that result from particular causes. This terminology arose from confusion in past GBD studies as to why something was a cause versus sequela versus risk factor. We do not use the term "surgical cause" in any of our definitions, as the definition of a surgical condition is based on a clinical problem rather than an established etiology. The latest round of the GBD study, funded by the Bill and Melinda Gates Foundation, is scheduled to be completed by November 2010. The final study is expected to produce specific DALY, YLL, and YLD estimates for more than 220 diseases/injuries and 40-plus risk factors by age range and sex for 21 regions of the world.

We recognize that there may be confusion about what constitutes surgical conditions and sequelae and that the availability of surgical providers may alter rates of surgical consultation. However, it should be feasible to define surgical conditions and sequelae using the International Classification of Disease (ICD) system. The ICD is a coding system of diseases and signs, symptoms, abnormal findings, complaints, social circumstances, and external causes of injury or diseases as well as therapeutic interventions, as classified by the World Health Organization (WHO) [12] As of 2008, the ICD-10 coding system includes 68,064 diagnoses and 86,917 procedure codes [13]. The increased detail in the ICD-10, compared to the ICD-9, should make it easier to reach a consensus on which codes represent surgical conditions.

Given the large number of diagnoses and procedures in ICD-10, it may be unrealistic to gather information (or estimate the burden) on the full spectrum of surgical conditions and/or interventions. A more practical approach might be to focus on a subset of these conditions or procedures. For example, the short list of "essential" surgical procedures (outlined by the WHO) could be expanded from facilities-based data gathered in low-income countries as well as published material. Procedures such as cesarean section, abscess drainage, laparotomy, and fracture care are likely to account for a significant percentage of interventions in most settings.

Recognizing that in many parts of the world surgical procedures are not done by fully trained surgeons, we use the term "surgically trained provider" rather than surgeon. In many other low-income countries, general practitioners may perform surgical procedures. Twenty-five Sub-Saharan countries utilize nonphysician clinicians, and almost half of them perform minor surgical procedures [14]. In Uganda, a study of five general hospitals reported that more than 5000 surgical procedures were performed annually by general practitioners [15]. With only nine orthopedic surgeons for than 20 million people, Malawi has trained orthopedic clinical officers to deliver most of the musculoskeletal services for the country [16]. In addition, $90 \%$ of the cesarean sections at the district hospital level in Malawi are carried out by surgically trained clinical officers [17]. In Mozambique, técnicos de cirurgia have performed major surgery in district hospitals since 1989 [18]. They perform 92\% of emergency obstetric care and $65 \%$ of major general surgery at the district hospital level. Other examples of surgically trained providers include nonphysician anesthesia providers [19]. A surgically trained provider implies a level of expertise in making a diagnosis, formulating a treatment plan (including the decision whether an operation is necessary), performing a procedure, and recognizing and treating any complications.

Our method for estimating the burden of surgical conditions and the unmet need for surgical care is based on the concept of "cumulative incidence." Cumulative incidence refers to the number of new cases that occur in a population over a period of time and is expressed in terms of the number of people at risk in the population at the beginning of the study. A key feature of cumulative incidence analysis is that an average age-specific cumulative risk curve can be plotted using population-based data. This is done under the assumption that age-specific incidence rates remain constant in the future. "Lifetime risk" of disease is a variation of the cumulative incidence concept and has been used by clinicians, researchers, and policymakers to assess the burden of a wide variety of diseases [20-26].

We use disability weights and values for surgical care to calculate cumulative surgical DALYs. This approach allows a DALY value to be assigned to each surgical condition and surgical intervention. In the case of rendered surgical care, the units of measure are expressed as DALYs averted. This approach is fundamentally the same as that used by McCord and Chowdhury [27] and Debas et al. [6], with the exception that their calculations were based on the 
percentage of averted risk. We believe that the "value of surgical care" concept allows a much more detailed analysis of surgical services. One concern is that it might be difficult to assign disability weights and values of surgical care to the large number of surgical conditions and interventions. Developing an approach that utilizes an average value for disability weight and value of surgical care could circumvent this problem.

The terminology we use to describe met and unmet need for surgical care is based on definitions used in obstetric need studies [28]. In these studies, obstetric need was estimated using census data, number of females at reproductive ages, and birth rates. Unfortunately, these methods are not as easily applied to the much broader spectrum of surgical conditions. Nevertheless, using terminology that is consistent with the obstetric literature should lessen any confusion.

An important feature of our proposed methodology is that the results will be comparable to other burden of disease studies. "DALYs" have been selected as the measure of burden of surgical conditions and "DALYs averted" as a measure of the impact of surgical care. This should be helpful in determining where surgical care fits among other global health priorities.

We recognize that the DALY continues to evolve in response to criticism and that this summary measure does not take into account contextual variables. Whereas the impairment associated with a particular disease process may be the same throughout the world, the disability associated with each condition will necessarily be determined by social and cultural variables in each environment. Also, disability weights have not yet been calculated for many of the surgical diseases. We suggest that research be undertaken to revise calculations for disability weights associated with surgical conditions, aimed at achieving a consensus opinion from health professionals (and possibly lay people) in both high- and low-income environments.

Finally, even with a strategy to measure the burden of surgical conditions and the unmet need for surgical care at the global level, there are still major obstacles to obtaining the needed estimates. Foremost is a profound lack of data on the incidence and prevalence of surgical conditions. This is especially the case in low- and middle-income countries, where hospital data are of limited value because of access problems and comprehensive surgical databases do not exist. Ideally, prospective community-based surveys will be undertaken in low- and middle-income countries to acquire the needed data.

\section{Conclusions}

We have described a strategy for measuring the impact of surgical care from a public health perspective. Using this methodology, it should be feasible to estimate the burden of surgical conditions and unmet need for surgery in a wide variety of populations.

Open Access This article is distributed under the terms of the Creative Commons Attribution Noncommercial License which permits any noncommercial use, distribution, and reproduction in any medium, provided the original author(s) and source are credited.

\section{Appendix 1}

Let the age groups be indexed by $i$, and the interval length and the rate of age-specific disability-adjusted life-years (DALYs) of each age group be represented by $n$ and $m$, respectively. In the absence of competing deaths, the cumulative surgical DALYs from birth to the end of the $i$ th age interval is given by the following equation:

Cumulative surgical DALYs $=\sum_{j \leq i}\left(n_{j} \times m_{j}\right)$.

\section{Appendix 2}

The burden of surgical conditions and unmet need for surgical care can be calculated from population-based data using basic epidemiologic data. Consider the bogus data set of surgical patients shown in Table 3. Included are data from ten patients from a population of 100 treated for surgical conditions during a 1-year period. The patient's age, sex, diagnoses, and treatment were arbitrarily selected to illustrate the utility of our method over a wide spectrum of patient ages and clinical conditions.

The age-specific values for years of life lost (YLL) are from Table 1.1 (Global Burden of Disease, or GBD, study) in ref. 29. Unless otherwise specified, disability weights for the surgical conditions are from Annex Table 3 (GBD study) in ref. 29. Disability weights of 1.0 were assigned to the patients with anorectal atresia, typhoid bowel perforation, strangulated inguinal hernia, and severe head injury under the assumption that these conditions would be fatal without surgical care.

The categories of the resulting DALYs (averted, potentially avertable, unavertable) were assigned by the surgical care/outcome. If patients' conditions were corrected by surgery, they were classified as "averted" and as "potentially avertable" if surgical care could have corrected the problem. The disability and death associated with severe head and spinal cord injury was classified as unavertable, as no care could have changed the outcome.

Table 4 summarizes data from the bogus data set. Data are categorized by the age groups used in the GBD study. We arbitrarily selected a population of 100 for our analysis and divided the population into different age groups using 
Table 3 Bogus data set of patients used to illustrate calculations of burden of surgical conditions and unmet need for surgical care $(n=10$ fictitious surgical patients)

\begin{tabular}{|c|c|c|c|c|c|c|c|c|c|}
\hline \multicolumn{4}{|c|}{ Patient characteristics } & \multicolumn{3}{|c|}{$\begin{array}{l}\text { Calculation of potential } \\
\text { DALYs }\end{array}$} & \multicolumn{3}{|c|}{ Category of DALYs ${ }^{\mathrm{d}}$} \\
\hline $\begin{array}{l}\text { Age } \\
\text { (years) }\end{array}$ & Sex & Condition & $\begin{array}{l}\text { Surgical care/ } \\
\text { outcome }\end{array}$ & $\mathrm{YLL}^{\mathrm{a}}$ & $\mathrm{DW}^{\mathrm{b}}$ & $\begin{array}{l}\text { Potential } \\
\text { DALYs }^{c}\end{array}$ & Averted & $\begin{array}{l}\text { Potentially } \\
\text { avertable }\end{array}$ & Unavertable \\
\hline 0 & Female & Cleft lip & Not repaired; survived & 33.12 & 0.098 & 3.2 & - & 3.2 & - \\
\hline 0 & Male & Anorectal atresia & Not repaired; died & 33.01 & 1 & 33.0 & - & 33 & - \\
\hline 10 & Female & $20 \%$ Burn, short term & Treated; survived & 37.62 & 0.186 & 7.0 & 7 & - & - \\
\hline 15 & Male & Typhoid bowel perforation & Not repaired; died & 36.8 & 1 & 36.8 & - & 36.8 & - \\
\hline 20 & Male & Strangulated inguinal hernia & Repaired; survived & 35.02 & 1 & 35.0 & 35 & - & - \\
\hline 25 & Male & Severe head injury & Treated; died & 32.53 & 1 & 32.5 & - & - & 32.5 \\
\hline 25 & Male & Spinal cord injury & Treated; paraplegic & 32.53 & 0.725 & 23.6 & - & - & 23.6 \\
\hline 30 & Female & Rectovaginal fistula & Repaired; survived & 29.92 & 0.43 & 12.9 & 12.9 & - & - \\
\hline 45 & Male & Gastric perforation & Repaired; survived & 20.17 & 1 & 20.2 & 20.2 & - & - \\
\hline 60 & Female & $\begin{array}{l}\text { Strangulated inguinal } \\
\text { hernia }\end{array}$ & Treated; survived & 12.22 & 1 & 12.2 & 12.2 & - & - \\
\hline Total & & & & & & 216.4 & 87.3 & 73.0 & 56.1 \\
\hline
\end{tabular}

$Y L L$ years of life lost, $D W$ disability weight

${ }^{a}$ Values are from Table 1.1 in ref. 29

b See text for how these values were assigned

${ }^{c}$ Potential surgical DALYS $=$ YLL $\times$ DW

${ }^{\mathrm{d}}$ Category of DALYs assigned by surgical care/outcome

Table 4 Calculating the burden of surgical conditions and unmet need for surgical care using population-based data

\begin{tabular}{|c|c|c|c|c|c|c|c|}
\hline \multirow[t]{2}{*}{ Parameter } & \multicolumn{5}{|c|}{ Age groups (years) } & \multirow[t]{2}{*}{ Total } & \\
\hline & $0-4$ & $5-14$ & $15-24$ & $25-59$ & $60+$ & & \\
\hline \multicolumn{8}{|l|}{ Raw data by age group ${ }^{a}$} \\
\hline No. of cases & 2.0 & 1.0 & 2.0 & 4.0 & 1.0 & \multicolumn{2}{|l|}{10.0} \\
\hline Population & 19.0 & 28.0 & 19.0 & 30.0 & 4.0 & \multicolumn{2}{|l|}{100.0} \\
\hline DALYs averted & 0.0 & 7.0 & 35.0 & 33.1 & 12.2 & \multicolumn{2}{|l|}{87.3} \\
\hline DALYs potentially avertable & 36.2 & 0.0 & 36.8 & 0.0 & 0.0 & \multicolumn{2}{|l|}{73.0} \\
\hline DALYs unavertable & 0.0 & 0.0 & 0.0 & 56.1 & 0.0 & \multicolumn{2}{|l|}{56.1} \\
\hline Total potential DALYs & 36.2 & 7.0 & 71.8 & 89.2 & 12.2 & \multicolumn{2}{|l|}{216.4} \\
\hline \multicolumn{8}{|c|}{ Age-specific incidence (DALYs $/ 10,000$ population/year) ${ }^{\mathrm{b}}$} \\
\hline DALYs averted & 0.0 & 70.0 & 350.0 & 94.6 & $58.1^{\mathrm{c}}$ & 572.7 & Met need ${ }^{\mathrm{d}}$ \\
\hline DALYs potentially avertable & 724.0 & 0.0 & 368.0 & 0.0 & 0.0 & 1092.0 & Unmet need $^{\mathrm{d}}$ \\
\hline DALYs unavertable & 0.0 & 0.0 & 0.0 & 160.3 & 0.0 & 160.3 & Unmeetable need $^{\mathrm{d}}$ \\
\hline Total potential DALYs & 724.0 & 70.0 & 718.0 & 254.9 & 58.1 & 1825.0 & Burden of surgical conditions $\mathrm{e}^{\mathrm{e}}$ \\
\hline
\end{tabular}

age distribution data for a population in Sub-Saharan Africa (Annex Table 11, GBD study [29]).

Age-specific incidence rates for averted, potentially avertable, unavertable, and total DALYs are shown in the lower half of Table 4. Incidence rates are expressed as DALYs/10,000 population/year. As described in Appendix A, age-specific incidence data can be used to calculate cumulative DALY curves. Note that the total sum total of 
the age group DALYs equals the cumulative surgical DALYs. Moreover, the values for cumulative DALYs (averted, potentially avertable, unmeetable, total) represent the met, unmet, and unmeetable need and the burden of surgical conditions.

\section{References}

1. PLoS Medicine Editors (2008) A crucial role for surgery in reaching the UN Millennium. Development Goals PLoS Med 5:1165-1167

2. Farmer P, Kim J (2008) Surgery and global health: a view from beyond the OR. World J Surg 32:533-536

3. Weiser TG, Regenbogen SE, Thompson KD et al (2008) An estimation of the global volume of surgery: a modelling strategy based on available data. Lancet 372:139-144

4. Ozgediz D, Jamison D, Cherian M et al (2008) The burden of surgical conditions and access to surgical care in low- and middle-income countries. Bull World Health Organ 86:646-647

5. Jamison D (ed) (2006) Disease control priorities in developing countries, 2nd edn. Oxford University Press, New York

6. Debas H, Gosselin R, McCord C (2006) Surgery. In: Jamison D et al (eds) Disease control priorities in developing countries. 2nd edition. Oxford University Press, New York

7. Global Burden of Surgical Disease Working Group (2009) http:// www.gsd2008.org/. Accessed 26 Aug 2009

8. Disease Control Priorities Project (2009) http://www.dcp2.org/ pubs/GBD. Accessed 26 Aug 2009

9. Bickler S, Spiegel D (2008) Global surgery-defining a research agenda. Lancet 12(372):90-92

10. Bickler SW, Telfer ML, Sanno-Duanda B (2008) Need for paediatric surgery care in an urban area of The Gambia. Trop Doct 33:91-94

11. Debas H, Jamison D, Lozano R et al (2009) Assessing the burden of surgical disease based on GBD 2005. BoSD Working Group Meeting, Chicago

12. WHO international classification of diseases (ICD). WHO, Geneva

13. American Health Information Management Association (AHIMA) (2009) http://www.ahima.org/icd10/faq.asp. Accessed 26 Aug 2009

14. Mullan F, Frehywot S (2007) Non-physician clinicians in 47 SubSaharan African countries. Lancet 370(9605):2158-2163

15. Ozgediz D, Galukande M, Mabweijano J et al (2008) The neglect of the global surgical workforce: experience and evidence from Uganda. World J Surg 32:1208-1215
16. Mkandawire N, Ngulube C, Lavy C (2008) Orthopaedic clinical officer program in Malawi: a model for providing orthopaedic care. Clin Orthop 466:2385-2391

17. Chilopora G, Pereira C, Kamwendo F et al (2007) Postoperative outcome of caesarean sections and other major emergency obstetric surgery by clinical officers and medical officers in Malawi. Hum Resource Health 14(5):17

18. Pereira C, Cumbi A, Malalane R et al (2007) Meeting the need for emergency obstetric care in Mozambique: work performance and histories of medical doctors and assistant medical officers trained for surgery. BJOG 114:1530-1533

19. Hodges SC, Mijumbi C, Okello M et al (2007) Anaesthesia services in developing countries: defining the problems. Anaesthesia 62:4-11

20. Lloyd-Jones DM, Larson MG, Beiser A et al (1999) Lifetime risk of developing coronary heart disease. Lancet 9(353):89-92

21. Garfinkel L (1995) Probability of developing or dying of cancer United States, 1991. Stat Bull Metropolitan Insurance Co 76:31-37

22. Schouten LJ, Straatman H, Kiemeney LA et al (1994) Cancer incidence: life table risk versus cumulative risk. J Epidemiol Community Health 48:596-600

23. Cummings SR, Black DM, Rubin SM (1989) Lifetime risks of hip, Colles', or vertebral fracture and coronary heart disease among white postmenopausal women. Arch Intern Med 149:2445-2448

24. Seshadri S, Wolf PA, Beiser A et al (1997) Lifetime risk of dementia and Alzheimer's disease: the impact of mortality on risk estimates in the Framingham Study. Neurology 49:1498-1504

25. Feuer EJ, Wung L, Boring CC et al (1993) The lifetime risk of developing breast cancer. J Natl Cancer Inst 85:892-897

26. Hopper JL, Southey MC, Dite GS et al (1999) Population-based estimate of the average age-specific cumulative risk of breast cancer for a defined set of protein-truncating mutations in BRCA1 and BRCA-2. Cancer Epidemiol Biomarkers Prev 8:741-747

27. McCord D, Chowdhury Q (2003) A cost-effective small hospital in Bangladesh: what it can mean for emergency obstetric care. Int J Gynaecol Obstet 81:83-92

28. Tackling unmet need for major obstetric interventions: Part 1 concepts, general principles and international network. UON network-unmet need for major obstetric interventions. http:// www.uonn.org/uonn/pdf/Guide1.pdf. Accessed 26 Aug 2009

29. Murray CJL, Lopez AD (1996) The global burden of disease: a comprehensive assessment of mortality and disability from diseases, Injuries, and risk factors in 1990 and projected to 2020. World Health Organization, Geneva 